\title{
AVALIAÇÃO TÉCNICA E ECONÔMICA DA EXTRAÇÃO DE MADEIRA DE EUCALIPTO COM “CLAMBUNK SKIDDER"
}

\author{
Robson José de Oliveira², Carlos Cardoso Machado ${ }^{3}$, Amaury Paulo de Souza ${ }^{3}$ e Helio Garcia Leite ${ }^{3}$
}

\begin{abstract}
RESUMO - O presente estudo teve como objetivo avaliar técnica e economicamente atividades de extração de madeira em vários declives, utilizando-se um trator florestal arrastador ("clambunk skidder") com rodado de pneus recoberto por esteiras. A avaliação técnica consistiu em determinar os índices de produtividade, e a avaliação econômica baseou-se na determinação dos custos operacionais da máquina. O delineamento experimental foi inteiramente casualizado, no esquema fatorial 3 × 2 × 5 (três níveis de declive, duas classes de produtividade florestal e cinco níveis de distâncias de extração com três repetições). Os resultados demonstraram que os estratos situados em menores declives e distâncias de extração apresentaram maior produtividade em relação àqueles de maiores declives e distâncias de extração. A produtividade do trator florestal arrastador avaliado variou entre $18,34 \mathrm{~m}^{3} \cdot \mathrm{h}^{-1} \mathrm{e} 72,08 \mathrm{~m}^{3} \cdot \mathrm{h}^{-1}$, com custo operacional médio de US $\$ 90,93$ por hora efetiva de trabalho.
\end{abstract}

Palavras-chave: Colheita, "clambunk skidder" e produtividade.

\section{TECHNICAL AND ECONOMIC EVALUATION OF EUCALYPTUS LOG EXTRACTION USING A CLAMBUNK SKIDDER}

\begin{abstract}
The objective of this study was to evaluate technical and economic log extraction activities using a clambunk skidder with tracks over the tires. The technical evaluation consisted in determining the log extraction productivity indexes and the economic evaluation was based on operational cost determination. The experimental design was complete randomized, with a $3 \times 2 \times 5$ factorial arrangement (three slope levels, two wood productivity classes and five ranks of extraction distance, with three repetitions). In a general way, the results showed that the equipment productivity decreases with the increase of the slope terrain and the extraction distance. Its productivity varied from $18.34 \mathrm{~m}^{3} \cdot \mathrm{h}^{-1}$ to $72.08 \mathrm{~m}^{3} \cdot \mathrm{h}^{-1}$. The operational cost was US\$ 90.93 per effective worked hour.
\end{abstract}

Keywords: Harvesting, clambunk skidder and productivity.

\section{INTRODUÇÃO}

Diversas empresas ainda utilizam métodos manuais e semimecanizados, apesar de toda a tecnologia à disposição para colheita florestal, em virtude de estarem localizadas em regiões de relevo desfavorável e também devido à disponibilidade de mão-de-obra. Aos poucos esse quadro vem diminuindo, porque esses métodos para colheita florestal se tornaram ultrapassados técnicas e economicamente, produzindo menores produtividades e encarecendo os custos operacionais (RESSEL FILHO, 2001).

A colheita de madeira necessita de bom planejamento, pois é uma atividade complexa por causa dos inúmeros fatores biológicos, ambientais, técnicos, humanos e

\footnotetext{
${ }^{1}$ Recebido em 15.09.2004 e aceito para publicação em 10.11.2005.

${ }^{2}$ Programa de Pós-Graduação em Ciência Florestal da Universidade Federal de Viçosa. E-mail: <engflorrobson@ vicosa.ufv.br>.

${ }_{3}^{3}$ Departamento de Engenharia Florestal da Universidade Federal de Viçosa. E-mail: <machado@ufv.br>.
} 
econômicos que a afetam, quer seja direta ou indiretamente (LOPES et al., 1999).

Devido à sua representatividade nos custos de produção, chegando a $50 \%$ do custo final da madeira posta na indústria a necessidade de mão-de-obra merece atenção especial por parte das empresas florestais, que têm investido em pesquisas visando a todo um processo de suprimento. Atualmente, a mecanização das atividades de colheita florestal tem sido uma prática usual na maioria das empresas florestais, que buscam a cada dia novas tecnologias e pesquisas, com o intuito de aumentar a produtividade e reduzir custos. Os equipamentos importados, por vezes tropicalizados, não conseguem atingir a mesma eficiência operacional de seus países de origem devido, principalmente, às diferenças edafoclimáticas de operação e à carência de pesquisas para a adaptação operacional desses equipamentos em nosso país (MACHADO, 1989).

A partir da década de 1990, a mecanização das operações de colheita e transporte florestal se intensificou no Brasil, com a abertura do mercado brasileiro à importação de máquinas e equipamentos desenvolvidos e aprimorados em países com maior tradição na colheita florestal (BRAMUCCI, 2001).

Hoje, com as empresas brasileiras investindo em pesquisas com o objetivo de melhorar a colheita florestal, por exemplo a busca de equipamentos e máquinas mais confortáveis e a realização de vários congressos e simpósios objetivando trocas de informações, estas vêm obtendo cada vez mais sucessos na implementação de sistemas mecanizados de colheita de madeira.

Com o objetivo de otimizar o máximo possível as operações, a colheita florestal vem sendo realizada pela maioria das empresas florestais com graus de mecanização distintos, em que, para descobrir o melhor sistema, estas ficam na dependência de uma série de fatores técnicos, humanos, ambientais, biológicos e econômicos (MACHADO et al., 2002).

A extração de madeira corresponde à movimentação de toretes por meio de arraste, suspensos por teleféricos ou em tratores autocarregáveis até a margem das estradas. Essa etapa exige um planejamento detalhado da operação, de maneira a empregar os equipamentos próprios dentro do sistema mais indicado de trabalho. Atualmente, no Brasil a extração mecanizada tem sido realizada por meio de "skidders" e "forwarders". O "skidder" surgiu

R. Árvore, Viçosa-MG, v.30, n.2, p.267-275, 2006 na década de 1960, sendo um veículo forte, fácil de operar e econômico; uma máquina que pode trabalhar com diferentes comprimentos de toretes. Trata-se de um trator florestal arrastador, desenvolvido exclusivamente para o arraste de madeira. Possui cabine com grande mobilidade dentro da área de corte, tem o chassi articulado, tração $4 X 4$, rodados de pneus revestidos por capa de esteiras. Alguns modelos são dotados de uma garra traseira e também telescópica, que é acionada pelo sistema hidráulico ou sistema de cabos de aço para que seja realizado o arraste de feixes de toretes. Existem três tipos básicos de skidders: com cabo, com garra e com garra e grua (MACHADO et al., 2002).

Segundo Partekforest (2003), as principais vantagens do "clambunk skidder" são:

a) A máquina-base pode voltar a operar como "forwarder".

b) Maior volume da madeira extraída por ciclo em relação ao "grapple skidder", permitindo operar em distâncias maiores.

c) Ccontribui para a redução da densidade de estradas florestais.

d) Pode operar inserido em vários sistemas de colheita, como: em conjunto com "harvester", "fellerbuncher", derrubada manual, garra traçadora, "slasher", "mini-slasher" e traçamento na estrada, entre outros.

e) Excelente do ponto de vista ergonômico.

f) Por ter pneus largos e, conseqüentemente, maior área de contato com o solo, tem-se melhor tração e estabilidade para operar em terrenos acidentados ou com baixa sustentação.

O principal objetivo deste trabalho foi analisar técnica e economicamente as atividades de extração de madeira de eucalipto em regiões acidentadas, utilizando-se o "clambunk skidder". Os objetivos específicos foram: determinar os custos operacionais da máquina; avaliar o desempenho técnico da máquina, determinando sua produtividade e potencialidade no sistema de árvores inteiras em diferentes condições de produtividade, declividade do terreno e distância de extração; analisar o custo de extração em diferentes classes operacionais; e identificar e avaliar os principais fatores que afetam a produtividade da máquina. 


\section{MATERIAL E MÉTODO}

\subsection{Area experimental}

O experimento foi conduzido em florestas comerciais de eucalipto, em duas áreas florestais na região do vale do Rio Doce, nos Municípios de Peçanha e Divinolândia de Minas, em áreas da Cenibra - Celulose Nipo-Brasileira S. A. Essa região tem latitude de $18^{\circ}$ 46' (S) e longitude de 42 $56^{\prime}$ (W), com relevo que varia de suave a forte ondulado, apresentando solos profundos, argilosos e férteis e precipitação anual em torno de $1.184 \mathrm{~mm}$ e temperatura do ar média de $27^{\circ} \mathrm{C}$.

\subsection{Sistema de colheita e máquinas utilizadas}

O sistema de colheita utilizado foi o de árvores inteiras com operações mecanizadas de corte, extração e traçamento. Na operação de corte foi utilizado um trator florestal derrubador-amontoador ("feller-buncher"), da marca Timberjack, modelo 608L, de esteiras e com cabine com nivelamento horizontal. Na extração de madeira foi utilizado um trator florestal dotado de rodado de pneus revestidos por capa de esteiras ("clambunk skidder"), conforme descrição a seguir.

Na operação de traçamento foi utilizado um conjunto carregador florestal com esteiras, marca Timberjack, modelo 635, sendo os toretes traçados no comprimento de $2,70 \mathrm{~m}$.

\subsection{Descrição do “clambunk skidder” e do ciclo operacional de trabalho}

O "clambunk skidder" utilizado é da marca Valmet, modelo 890.1, tração 8X8, de pneus revestidos por capa de esteiras (Figura 1). Possui uma garra marca Cranab G36, com área útil de 1,82 $\mathrm{m}^{2}$. Possui tanque com capacidade para $206 \mathrm{~L}$ de óleo diesel e um tanque de óleo do sistema hidráulico com capacidade para 145 L. A capacidade de carga da máquina é de $176.600 \mathrm{~N}$.

O modelo do motor da máquina é Valmet 634 DWBIE, de seis cilindros, turbo alimentado com $154 \mathrm{~kW}$ de potência bruta, torque de $885 \mathrm{mN}$ a $1.200 \mathrm{rpm}$ e um consumo de combustível da ordem de 16,5 1/h (PARTEKFOREST, 2003).

O "clambunk skidder" realizou o ciclo operacional da seguinte forma:

a) Deslocamento - Começa quando o "clambunk skidder" sai da margem da estrada sem carregamento de madeira, iniciando a etapa da viagem sem carga em direção aos feixes de toretes no interior do talhão.

b) Carregamento - Inicia-se com a abertura dos arcos da grua, levantando e carregando os toretes e acondicionando-os dentro da garra e terminando quando a garra está com carga completa.

c) Arraste - Começa quando a máquina inicia o deslocamento com a carga parcialmente suspensa, em direção à margem da estrada.

d) Descarregamento - Ao se aproximar da margem da estrada, o operador descarrega os feixes, depositandoos de forma que não comprometam a etapa seguinte da colheita florestal.

O "clambunk skidder" possui uma grande vantagem, que é o fato de vir até a margem da estrada, até o talude, descarregar as árvores e voltar de ré, como mostra a Figura 1 o momento em que a máquina acaba de depositar o feixe de toretes na margem da estrada e se prepara para iniciar um novo ciclo de operação, com deslocamento vazio.

\subsection{Instrumental de medição}

Por meio de um clinômetro modelo Timbco Hydraulic LLC P/N 24198 usado para determinar o declive do terreno e de um computador de bordo marca Maxi Forwarder, que se encontram instalados na máquina, podem ser feitas as leituras de distância de extração, temperatura do óleo do motor, conta-giros e velocidade, entre outros parâmetros associados a um cronômetro que disparava a cada início de ciclo operacional.

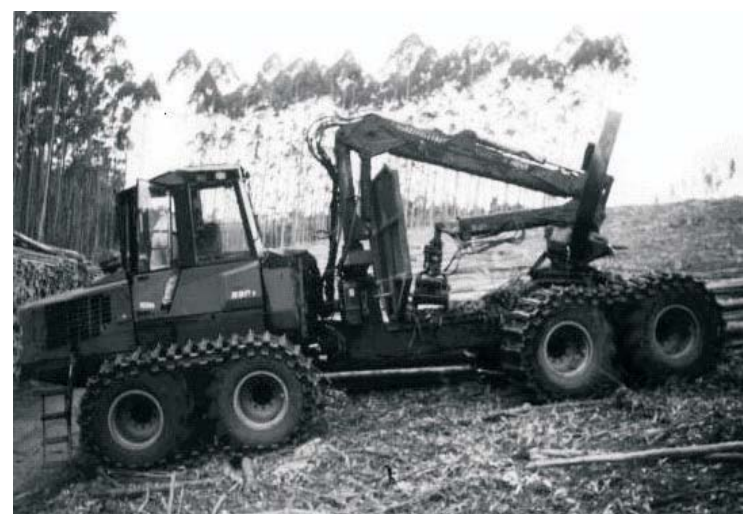

Figura 1 - "Clambunk skidder" em operação.

Figure 1 - Clambunk skidder in operation.

R. Árvore, Viçosa-MG, v.30, n.2, p.267-275, 2006 
O estudo de tempos e movimentos foi realizado pelo método de tempos contínuos, em que se mediu o tempo total do ciclo do "clambunk skidder", anotando as distâncias e declives, em que eram arrastados os feixes até a margem da estrada. Foi realizado previamente um estudo-piloto, em que foram feitas cinco tomadas de tempo do ciclo operacional, visando estabelecer quantas repetições eram necessárias e, com isso, foram realizadas três repetições para cada distância, declividade e área de produção.

\subsection{Delineamento experimental}

O delineamento utilizado foi inteiramente casualizado no esquema fatorial $3 \times 2 \times 5$ (três níveis de declive, duas classes de produtividade da floresta e cinco níveis de distâncias de extração com três repetições), resultando em 90 ciclos operacionais, sendo:

a) Classes de produtividade da floresta no momento da colheita:

a.1) Floresta I: $<350 \mathrm{~m}^{3} / \mathrm{ha}$

a.2) Floresta II: $>350 \mathrm{~m}^{3} / \mathrm{ha}$

b) Classes de distância de extração:

b.1) Distância I: $0 \mathrm{~m}$ a $50 \mathrm{~m}$

b.2) Distância II: 50 m a $100 \mathrm{~m}$

b.3) Distância III: 100 m a 150 m

b.4) Distância IV: 150 m a 200 m

b.5) Distância V: 200 m a 250 m.

c) Classes de declividade média da área

c.1) Suave: $0^{\circ}$ a $9^{\circ}$

c. 2) Ondulada: $9^{\circ}$ a $18^{\circ}$

c.3) Acidentada: $18^{\circ}$ a $27^{\circ}$.

Foram ajustados modelos de regressão para avaliar o efeito da distância de extração, do declive e da produtividade da floresta sobre a produtividade da máquina. Foram consideradas como melhores equações aquelas com maior coeficiente de determinação e melhor distribuição dos resíduos.

\subsection{Avaliação econômica}

$\mathrm{Na}$ determinação dos custos de produção, considerou-se o tempo total de trabalho, ou seja, não foram analisadas separadamente as etapas do ciclo

R. Árvore, Viçosa-MG, v.30, n.2, p.267-275, 2006 operacional da máquina. Para o cálculo dos custos operacionais foi utilizada uma planilha fornecida pela empresa executora que considera os custos fixos e variáveis descritos a seguir, contendo, ainda, informações sobre a disponibilidade mecânica e eficiência operacional. Os dados foram analisados em um programa estatístico denominado curve expert 1.3 , chegando a equações que melhor representam os dados ajustados às curvas a modelos que melhor explicam os comportamentos, depois de determinados os custos operacionais.

\subsection{Determinação de Custos Operacionais}

Para determinação dos custos operacionais, utilizouse o método contábil com valores estimados e reais. Os custos fixos foram avaliados pela metodologia proposta pela FAO (1974), citada por Machado e Malinovski (1988), enquanto para os custos variáveis se utilizaram dados fornecidos pela empresa executora.

\subsubsection{Custos Fixos}

a) Depreciação (Dp)

A depreciação da máquina está associada a desgaste natural através do tempo de uso, sendo isso um modo de recuperar o seu investimento original. A inclusão do seu valor no custo operacional representa a constituição de um capital de reserva para aquisição de uma nova máquina. Para cálculo da depreciação foi utilizado o método linear:

$$
D p: \frac{V a-V r}{N^{*} h e}
$$

em que:

$\mathrm{DP}=$ depreciação linear da máquina (US\$/he);

$\mathrm{Va}=$ valor de aquisição da máquina (US\$);

$\mathrm{Vr}=$ valor residual da máquina (US\$);

$\mathrm{N}=$ vida útil estimada (anos); e

he $=$ horas efetivas de uso anual.

b) Juros (J)

Os juros foram calculados pela aplicação de uma taxa ao investimento, correspondente ao capital proporcionado por agência financeira:

$$
J=\frac{(C a \times i)}{V u}
$$


em que:

$$
\begin{aligned}
& \mathrm{J}=\text { juros (US\$/he); } \\
& \mathrm{Ca}=\text { custo de aquisição da máquina (US\$); } \\
& \mathrm{i}=\text { taxa anual de juros }(\%) ; \mathrm{e} \\
& \mathrm{Vu}=\text { vida útil da máquina (horas). }
\end{aligned}
$$

\subsubsection{Custos Variáveis (CV)}

São os custos proporcionais à quantidade de madeira produzida, ou seja, com a intensidade de uso da máquina. São constituídos pelos custos de combustível, lubrificantes, pneus/esteira, remuneração de pessoal e manutenção/reparos ou peças.

a) Custo de combustível (CC)

É o custo referente ao consumo de óleo diesel, calculado pela fórmula:

$$
C C=P u \times c
$$

em que:

CC = custo de combustível (US\$/he);

$\mathrm{Pu}=$ preço de $1 \mathrm{~L}$ de óleo diesel (US\$/L); e

$\mathrm{C}=$ consumo de óleo diesel por hora efetiva de trabalho (1/he).

\section{b) Custo de lubrificantes e graxas (CLG)}

Refere-se ao consumo de óleos lubrificantes e graxas. Considerou-se que esses custos equivalem a um índice igual a 54\% do valor gasto com combustível.

$$
C L G=I \lg x C c
$$

em que:

$\mathrm{CLG}=$ custo com lubrificantes e graxas (US\$/he);

I lg = índice de custos com lubrificantes e graxas; e

$\mathrm{Cc}=$ custos com combustível (US\$/he).

c) Custo de pneus/esteiras (CPE)

Representa o custo referente aos gastos com a manutenção de pneus e esteiras. Esse custo é influenciado pelas condições do terreno, ambiente, manutenção do rodado, habilidade e cuidados do operador.

$$
C P E=\frac{N p e \times V p e}{H}
$$

em que:

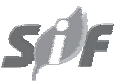

CPE = custo de manutenção do conjunto pneus/ esteiras (US\$/he);

Npe $=$ número de conjuntos de pneus/esteiras por máquina;

$\mathrm{Vpe}=$ valor de um conjunto pneu/esteira da máquina (US\$/unidade); e

$\mathrm{H}=$ vida útil do pneu/esteira, em horas efetivas.

d) Custo de pessoal operacional (COM)

Refere-se a salários diretos, benefícios e encargos sociais, como décimo-terceiro salário, férias, seguros, assistência médica e previdenciária, EPIS, alimentação, vestuário, custo referido a valores mensais divididos pelo número de horas trabalhadas por mês.

e) Custo com manutenção e reparos (CMR)

Refere-se ao custo relacionado à manutenção, peças e reparos mecânicos a que as máquinas estão sujeitas durante sua vida útil. Envolve os gastos com terceiros, oficina, comboio de abastecimento e lubrificação.

\subsubsection{Custos de administração (CAD)}

Representa os gastos relacionados aos trabalhos de escritório e supervisão das atividades de campo, os quais também foram obtidos na empresa onde se realizou o trabalho, nesse caso considerando-se esses gastos como $10 \%$ dos custos totais em valores mensais e divididos pelo número de horas trabalhadas por mês.

\subsubsection{Custo operacional total (CT)}

Este custo foi obtido pela soma dos gastos fixos, variáveis e custos de administração relativos à máquina analisada neste trabalho, ou seja:

$$
\mathrm{CT}=\mathrm{CF}+\mathrm{CV}+\mathrm{CAD}
$$

em que:

$\mathrm{CT}=$ custo operacional total da máquina (US\$/he);

$\mathrm{CF}=$ custos fixos (US\$/he);

$\mathrm{CV}=$ custos variáveis (US\$/he); e

$\mathrm{CAD}=$ custos de administração (US\$/he).

\subsection{Produtividade da máquina}

A produtividade do "clambunk skidder" foi estimada segundo as variáveis da matriz de amostragem, considerando-se o volume máximo do feixe de $4,91 \mathrm{~m}^{3}$.

R. Árvore, Viçosa-MG, v.30, n.2, p.267-275, 2006 


\subsection{Custo de extração}

O custo de extração foi obtido pela seguinte fórmula:

$$
C E=\frac{C T}{\operatorname{Pr} o d}
$$

em que:

$\mathrm{CE}=$ custo de extração do "clambunk skidder" $\left(\mathrm{US} \$ / \mathrm{m}^{3}\right)$;

CT $=$ custo operacional total do "clambunk skidder" (US\$/h); e

Prod $=$ produtividade do "clambunk skidder" $\left(\mathrm{m}^{3} / \mathrm{h}\right)$.

\subsection{Disponibilidade mecânica e eficiência operacional}

A disponibilidade mecânica é definida como a porcentagem do tempo de serviço programado em que a máquina está mecanicamente apta a realizar trabalho produtivo, desconsiderando-se, portanto, o tempo em que a máquina está em conserto ou manutenção. Pode ser expressa pela seguinte expressão:

$$
D M=\frac{H E}{(H E+T P M)} \times 100
$$

em que:

DM = grau de disponibilidade mecânica (\%);

$\mathrm{HE}=$ tempo de trabalho efetivo (horas); e

TPM = tempo de permanência em manutenção (horas).

A eficiência operacional é definida como a porcentagem do tempo efetivamente trabalhado, em relação ao tempo total programado para o serviço. Pode ser expressa pela seguinte expressão:

$$
E O=\frac{H E}{(H E+H P)} \times 100
$$

em que:

$\mathrm{EQ}=$ eficiência operacional (\%);

$\mathrm{HE}=$ tempo de trabalho efetivo (horas); $\mathrm{e}$

$\mathrm{HP}=$ horas paradas (horas)

A empresa realiza a manutenção preventiva nas máquinas para garantir a disponibilidade mecânica previamente estabelecida. Com base em dados reais contábeis retirados de uma planilha fornecida pela empresa executora e na utilização das fórmulas anteriormente citadas, obtém-se o valor de $74 \%$ para disponibilidade mecânica e $83 \%$ para eficiência operacional.

\section{RESULTADOS E DISCUSSÃO}

\subsection{Estimativa do custo operacional do "Clambunk skidder"}

Na estimativa do custo operacional, utilizou-se a taxa de câmbio de 1 dólar $=\mathrm{R} \$ 2,9540$ e 1 euro $=\mathrm{R} \$$ $3,5619(30 / 08 / 2004)$.

A taxa anual de juros de $12 \%$, o valor residual da máquina de $20 \%$ e uma taxa de administração de $10 \%$ dos custos totais resultaram em um custo por hora efetiva de trabalho de US\$ 90,93/he.

A distribuição espacial dos elementos do custo operacional é mostrada na Figura 2. Os custos variáveis diretos (combustível, lubrificantes, peças e pneus/esteira) totalizaram $47 \%$, ou seja, cerca da metade dos gastos operacionais, seguidos dos custos fixos (depreciação e juros), que corresponderam a $37 \%$ dos custos operacionais, seguidos dos custos variáveis indiretos (comboio, salários e terceiros), com $16 \%$.

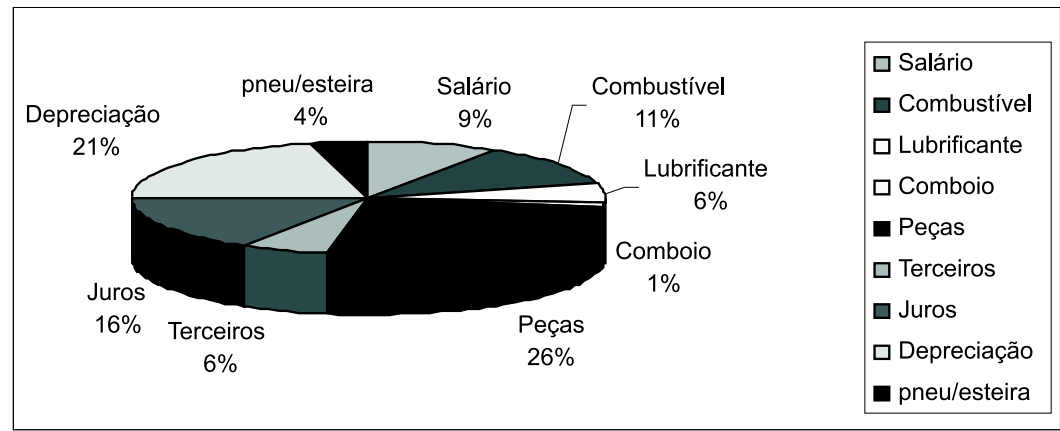

Figura 2 - Distribuição dos custos operacionais do "clambunk skidder".

Figure 2 - Operational costs distribution of clambunk skidder. 


\subsection{Estimativa de tempos consumidos na extração de madeira}

As equações de tempos consumidos na extração da madeira foram geradas em função das distâncias de extração, utilizando-se um programa estatístico denominado curve expert (Quadro 1).

Pelas equações do Quadro 1, para cada classe de floresta e declividades obtêm-se os dados de tempo que são transformados de segundo para hora, dividindo por 3600 para calcular a produtividade e pegando o volume máximo do feixe de $4,91 \mathrm{~m}^{3}$, dividindo pelo resultado citado anteriormente e chegando à produtividade, como ilustrado na Figura 3.

Analisando a Figura 3, observa-se na classe de distância de extração até $100 \mathrm{~m}$ que a produtividade do "Clambunk skidder" foi a mais elevada na classe de floresta 2 ( $>350 \mathrm{~m}^{3} / \mathrm{ha}$ ) e declividade suave ( 0 a $9^{\circ}$ ). Entre as distâncias de 100 a 250 m, o melhor desempenho, em termos de produtividade, foram floresta $1\left(<350 \mathrm{~m}^{3} / \mathrm{ha}\right)$ e declividade ondulada $\left(9\right.$ a $\left.18^{\circ}\right)$. Na classe de distância de extração até $100 \mathrm{~m}$, verificouse que as produtividades mais baixas ocorreram na floresta $2\left(>350 \mathrm{~m}^{3} / \mathrm{ha}\right)$ e na declividade ondulada ( 9 a $\left.18^{\circ}\right)$. O mesmo aconteceu com as distâncias superiores a $100 \mathrm{~m}$, na floresta $2\left(>350 \mathrm{~m}^{3} / \mathrm{ha}\right)$ e na declividade acidentada $\left(18\right.$ a $\left.27^{\circ}\right)$. Houve influência da galhada presente no solo, o que causou empecilho ao deslocamento da máquina, ocorrendo aumento sistemático no tempo do ciclo operacional, em algumas classes de declividade dentro de um tipo de floresta, em comparação com outros tempos nas demais classes de declividade, traduzindo em maiores dificuldades operacionais para a máquina e, conseqüentemente, menores produtividade e maior custo de extração.

\subsection{Determinação do custo de extração}

Na Figura 4, apresenta-se uma curva do tipo exponencial relacionando o custo de extração em função da produtividade do "Clambunk skidder". Com o resultado da produtividade, o custo de extração é calculado com base no custo por hora efetiva de trabalho dividido pelas produtividades. O custo variou de US\$ 4,96/ $\mathrm{m}^{3}$ na faixa de maior distância de extração e declividade do terreno a US $\$ 1,26 / \mathrm{m}^{3}$, na faixa de melhores condições de operação. Foi traçada uma linha de tendência que gerou a equação representada na Figura 4.

Quadro 1 - Equações de tempos operacionais consumidos em função de diferentes distâncias de extração Table 1 - Time of log extraction as a function of different distances

\begin{tabular}{|c|c|c|c|}
\hline F/D & Classe de floresta & Classe de declividade & Equação \\
\hline F1/D1 & $\left(<350 \mathrm{~m}^{3} / \mathrm{ha}\right)$ & $\left(<9^{\circ}\right)$ & $Y=452,16662 *(1-\exp (-0,020515332 * D E))$ \\
\hline F1/D2 & $\left(<350 \mathrm{~m}^{3} / \mathrm{ha}\right)$ & $\left(10^{\circ}\right.$ a $\left.18^{\circ}\right)$ & $Y=-7,05143 *(-43,829777-\exp (-0,016629852 * D E))$ \\
\hline F1/D3 & $\left(<350 \mathrm{~m}^{3} / \mathrm{ha}\right)$ & $\left(19^{\circ}\right.$ a $\left.27^{\circ}\right)$ & $\mathrm{Y}=218,2631 * \exp (0,0047558563 * \mathrm{DE})$ \\
\hline F2/D1 & $\left(>350 \mathrm{~m}^{3} / \mathrm{ha}\right)$ & $\left(<9^{\circ}\right)$ & $\mathrm{Y}=1245,963 *(1-\exp (-0,0043836854 * \mathrm{DE}))$ \\
\hline $\mathrm{F} 2 / \mathrm{D} 2$ & $\left(>350 \mathrm{~m}^{3} / \mathrm{ha}\right)$ & $\left(10^{\circ}\right.$ a $\left.18^{\circ}\right)$ & $\mathrm{Y}=465,8+(0,33542857 * \mathrm{DE})+0,00662857 *(\mathrm{DE})^{2}$ \\
\hline $\mathrm{F} 2 / \mathrm{D} 3$ & $\left(>350 \mathrm{~m}^{3} / \mathrm{ha}\right)$ & $\left(19^{\circ}\right.$ a $\left.27^{\circ}\right)$ & $\mathrm{Y}=1158,1816 * \operatorname{EXP}(-57,902117 / \mathrm{DE})$ \\
\hline
\end{tabular}

$\mathrm{F} / \mathrm{D}=$ classe de produtividade da floresta/classe de declividade. $\mathrm{F} 1 \mathrm{D} 1$ = floresta 1 e declividade $1 . \mathrm{F} 1 \mathrm{D} 2$ = floresta 1 e declividade 2. F1D3 = floresta $1 \mathrm{e} \mathrm{declividade} 3$. F2D1 = floresta $2 \mathrm{e}$ declividade 1. F2D2 = floresta $2 \mathrm{e}$ declividade 2. F2D $3=$ floresta 2 e declividade 3 $\mathrm{Y}=$ tempo $(\mathrm{s}) . \mathrm{DE}=$ distância de extração $(\mathrm{m})$.

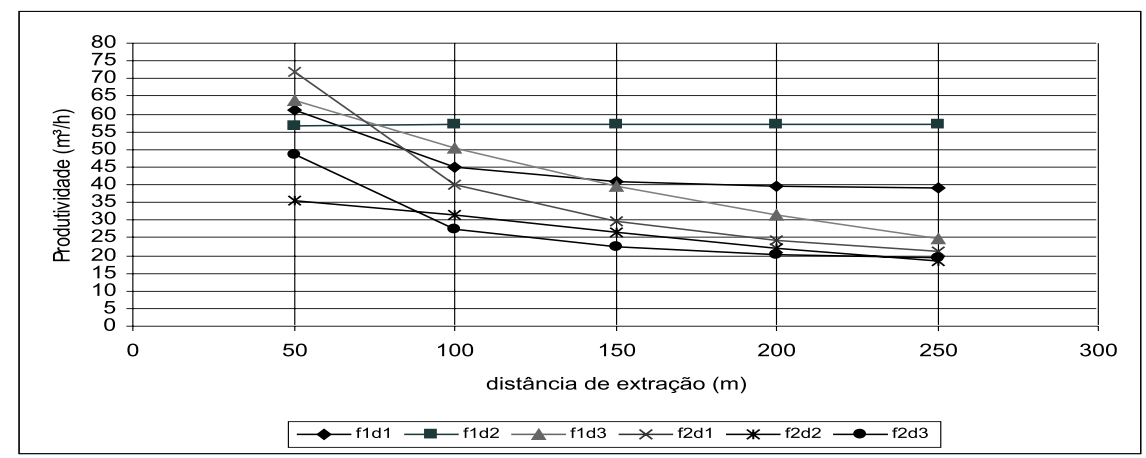

Figura 3 - Produtividade média de arraste em função da distância de extração.

Figure 3 - Average skidding productivity as function of the extraction distance. 


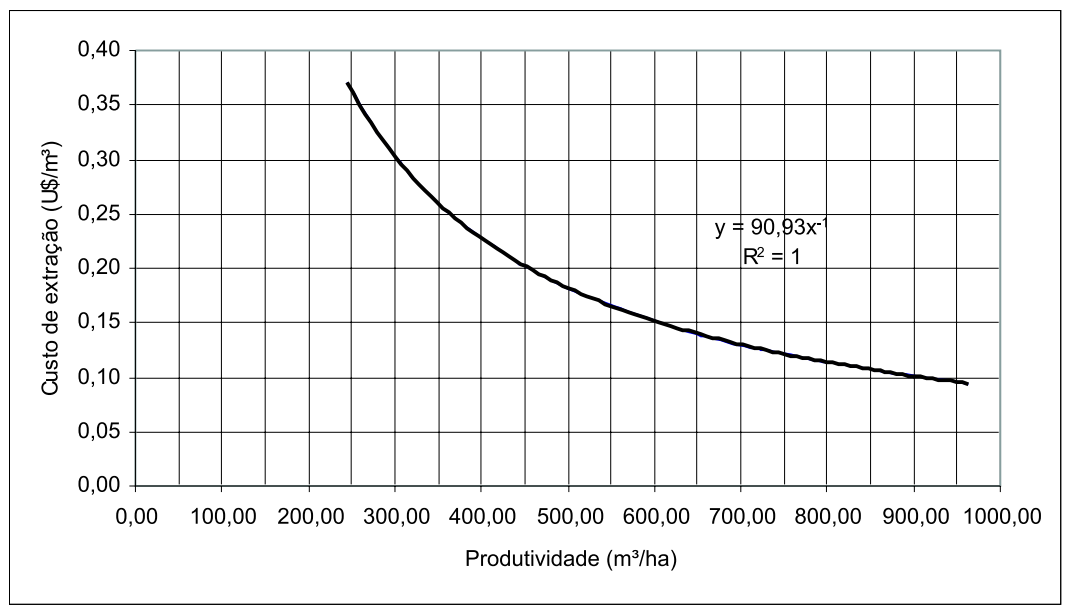

Figura 4 - Comportamento do custo de extração de madeira em função da produtividade. Figure 4-Wood extraction cost behavior as a function of productivity.

\subsection{Avaliação das características operacionais e fatores que afetam a extração}

A declividade e a distância de extração são fatores que afetam significativamente o trabalho do "clambunk skidder", como visto na Figura 3. Quanto mais acidentado o relevo e mais distantes os feixes para o arraste, menor a produtividade, elevando-se os custos de extração nesses locais. Outros fatores, como volume e rendimento da máquina, clima, precipitação e treinamento dos operadores, influenciam também não só a extração, mas sim a atividade florestal como um todo.

\section{CONCLUSÕES}

As principais conclusões deste estudo foram:

a) A produtividade do trator florestal arrastador avaliado variou entre $18,34 \mathrm{~m}^{3} \cdot \mathrm{h}^{-1} \mathrm{e} 72,08 \mathrm{~m}^{3} \cdot \mathrm{h}^{-1}$, com custo operacional médio de US\$ 90,93 por hora efetiva de trabalho.

b) Na classe de maior distância de extração (201$250 \mathrm{~m}$ ), a produtividade do "clambunk skidder" foi maior na declividade ondulada (10 a $\left.18^{\circ}\right)$ para floresta $1(<$ $\left.350 \mathrm{~m}^{3} / \mathrm{ha}\right)$ e declividade suave $\left(0\right.$ a $\left.9^{\circ}\right)$ para floresta $2\left(>350 \mathrm{~m}^{3} / \mathrm{ha}\right)$.

c) O custo de extração mais baixo na classe de distância de extração (0-50 m) ocorreu na floresta de classe $2(>350 \mathrm{~m} / \mathrm{ha})$ e na declividade suave $\left(0\right.$ a $\left.9^{\circ}\right)$. Na classe de distância de extração maior (201-250 m), os custos menores ocorreram na floresta de classe 1 $\left(<350 \mathrm{~m}^{3} / \mathrm{ha}\right)$ e declividade ondulada $\left(10\right.$ a $\left.18^{\circ}\right)$.

d) Em curtas distâncias de extração, em declividade ondulada ( 10 a $\left.18^{\circ}\right)$ e na classe de floresta $1\left(<350 \mathrm{~m}^{3} /\right.$ ha), o equipamento produziu quase $60 \%$ a mais do que na classe de floresta $2\left(>350 \mathrm{~m}^{3} / \mathrm{ha}\right)$.

\section{AGRADECIMENTOS}

À Coordenação de Aperfeiçoamento de Pessoal de Nível Superior (CAPES) e à CENIBRA, pelo apoio.

\section{REFERÊNCIAS BIBLIOGRÁFICAS}

BRAMUCCI, M. Determinação e quantificação de fatores de influência sobre a produtividade de "harvesters" na colheita de madeira. 2001. 50f.

Dissertação (Mestrado em Recursos florestais) Escola Superior de Agricultura Luiz de Queiroz, Piracicaba, 2001.

FOOD AND AGRICULTURALORGANIZATIONFAO. Logging and log transport in manmade forests in developing countries. Roma: 1974. 90p. (Forest Paper - FAO,18).

LOPES, E. S. et al. Influência de alguns aspectos ergonômicos e sociais no planejamento da colheita florestal. In: SIMPÓSIO BRASILEIRO SOBRE COLHEITAE TRANSPORTE FLORESTAL, 4., 1999, Campinas. Anais... Viçosa, MG: Sociedade de Investigações Florestais, 1999. p.322-330. 
MACHADO, C.C. Exploração florestal, Viçosa, MG: Universidade Federal de Viçosa, 1989. v.6. 34p.

MACHADO, C.C.; MALINOVSKI, J.R. Ciência do trabalho florestal. Viçosa, MG:

Universidade Federal de Viçosa, 1988. 65p.

MACHADO, C.C. et al. Análise de custos de subsistemas de colheita de toras de eucalipto de diferentes comprimentos e volumes por árvore.

Revista Árvore, v.26, n.1, p.73-81, 2002.
PARTEKFOREST. Disponível em: $<\mathbf{h t t p}: / /$ www.partekforest.com>. Acesso em: 10 Dez. 2003.

RESSEL FILHO, E.H. Rendimento da colheita semimecanizada e extração de madeira em $1^{\circ}$ desbaste de eucalyptus grandis Hill ex maiden na Klabin Riocell, em Guaíba/RS. In: SIMPÓSIO BRASILEIRO SOBRE COLHEITAE TRANSPORTE FLORESTAL, 5., 2001, Porto Seguro. Anais... Viçosa, MG: Sociedade de Investigações Florestais, 2001. p.194-205. 